\title{
Escalas de exploração vocacional para estudantes de ensino médio
}

\author{
Vocational exploration scales \\ for high school students
}

\author{
Marco Antônio Pereira TEIXEIRA ${ }^{1}$ \\ Ana Cristina Garcia DIAS
}

\begin{abstract}
Resumo
A exploração vocacional é essencial para o desenvolvimento vocacional e para as tomadas de decisão vocacionais. O objetivo deste estudo foi testar as propriedades psicométricas de uma versão adaptada para estudantes do ensino médio das Escalas de Exploração Vocacional, que avaliam duas dimensões de exploração vocacional: exploração de si e do ambiente. As escalas foram aplicadas a 436 estudantes de ensino médio (média de idade de 16,3 anos; 55,3\% mulheres). Análises de componentes principais revelaram os dois componentes esperados, sugerindo a validade de construto do instrumento. Os índices de fidedignidade obtidos também foram satisfatórios. Conclui-se que as Escalas de Exploração Vocacional são instrumentos válidos e fidedignos para avaliar exploração vocacional, podendo ser utilizadas em futuras pesquisas.
\end{abstract}

Unitermos: Avaliação psicológica. Comportamento exploratório. Exploração de carreira. Exploração vocacional.

\begin{abstract}
Vocational exploration is essential to vocational development and for career decision-making. The aim of this study was to test the psychometric properties of a version of the Vocational Exploration Scales adapted for use with high school students, which evaluate two dimensions of vocational exploration: self-exploration and environmental exploration. The scales were applied to 436 high school students (mean age: 16.3 years; $55.3 \%$ women). Principal components analyses revealed the two expected components, suggesting the construct validity of the instrument. Reliability indices obtained were also satisfactory. It may be concluded that the Vocational Exploration Scales are valid and reliable instruments for assessing vocational exploration that can be used in future research.
\end{abstract}

Uniterms: Psychological assessment. Exploratory behavior. Career exploration. Vocational exploration.

O comportamento exploratório é um aspecto central no desenvolvimento vocacional e nos processos de tomada de decisão de carreira (Jordaan, 1963; D. E. Super, Savickas \& C. M. Super, 1996). É por meio da exploração que o indivíduo reflete sobre si mesmo e sobre o mundo do trabalho, obtendo e organizando informações importantes para suas escolhas vocacionais (Flum \& Blustein, 2000; Taveira, 2000).

O conceito de comportamento exploratório vocacional foi inicialmente desenvolvido por Jordaan

$\boldsymbol{\nabla \nabla \boldsymbol { \nabla }}$

1 Universidade Federal do Rio Grande do Sul, Instituto de Psicologia, Programa de Pós-Graduação em Psicologia. R. Ramiro Barcelos, 2600, Sala 117, 90035-003, Porto Alegre, RS, Brasil. Correspondência para/Correspondence to: M.A.P. TEIXEIRA. E-mail: <mapteixeira@yahoo.com.br>.

2 Universidade Federal de Santa Maria, Departamento de Psicologia. Santa Maria, RS, Brasil. 
(1963), que descreveu esse comportamento como parte de um processo de resolução de problemas. Diante de uma situação ambígua ou de crise, que exige uma decisão ou escolha, o indivíduo aumentaria seus comportamentos exploratórios a fim de obter informações suficientes para decidir-se por um curso de ação. Assim, comportamentos exploratórios ocorreriam durante toda a vida, mas seriam mais salientes em momentos de transição nos quais o indivíduo se vê compelido a tomar decisões importantes e percebe ser necessário obter informações que o auxiliem nesse processo (Jordaan, 1963).

Super (1963) caracterizou a exploração como um estágio dentro do desenvolvimento vocacional que corresponderia aos anos da adolescência e do início da vida adulta, período no qual a tarefa evolutiva de escolher uma ocupação exigiria do sujeito um aumento nos comportamentos exploratórios. Essa exploração se daria em duas grandes dimensões: uma voltada para o interior do indivíduo (self-exploration, ou exploração de si) e outra para o exterior, ou mundo ocupacional (environmental exploration, ou exploração do ambiente). Essas dimensões estariam correlacionadas, pois à medida que o indivíduo interroga a si mesmo sobre seus interesses e valores (exploração de si) surge a necessidade de conhecer o mundo educacional e ocupacional (exploração do ambiente). Por sua vez, o conhecimento das possibilidades existentes faz com que o indivíduo precise refinar suas autoavaliações a fim de poder fazer escolhas que correspondam com maior grau de especificidade às suas características, interesses e valores pessoais. Estudos empíricos têm comprovado a existência de correlação entre essas dimensões (Sparta, 2003; Stumpf, Colarelli \& Hartmann, 1983).

Atualmente, a exploração vocacional é vista mais como um processo que ocorre durante toda a vida do que como apenas uma etapa circunscrita no tempo (Flum \& Blustein, 2000; Super et al., 1996). Sua função é eminentemente adaptativa para o sujeito, pois quanto maior a clareza que um indivíduo tem a respeito de si mesmo e da realidade educacional e do trabalho, mais bem preparado ele estará para se orientar em um mundo em constante mudança.

A importância do comportamento exploratório no âmbito das teorias de desenvolvimento vocacional 90 e de aconselhamento de carreira se evidencia pela diversidade de pesquisas que incluem a avaliação da exploração vocacional, relacionando-a com outras variáveis. Por exemplo, há estudos que mostram existir associação entre exploração e decisão de carreira (Sparta, 2003; Taveira, 2000), autoeficácia vocacional (Rogers, Creed \& Glendon, 2008), características de personalidade (Reed, Bruch \& Haase, 2004; Rogers et al., 2008), estabelecimento na carreira (Jepsen \& Dickson, 2003) e nível de identidade vocacional (Scott \& Ciani, 2008). Além disso, um dos focos nas intervenções de aconselhamento de carreira são atividades que buscam levar os indivíduos a explorar suas possibilidades educacionais e ocupacionais como forma de aumentar sua capacidade de tomar decisões (Hirschi \& Läge, 2008).

No Brasil, foram localizados poucos estudos que tratam da avaliação do comportamento exploratório vocacional com população adolescente. Em geral, a exploração é avaliada qualitativamente, como um elemento presente no processo de escolha profissional (Magalhães, Lassance \& Gomes, 1998). Apenas duas pesquisas com instrumentos específicos que medem o construto de exploração em adolescentes foram identificadas: os trabalhos de Frischenbruder (1999) e Sparta (2003), que buscaram adaptar o instrumento Career Exploration Survey (CES) (Stumpf et al., 1983) para uso com adolescentes brasileiros. O CES é um instrumento muito utilizado internacionalmente nas pesquisas em desenvolvimento vocacional, e inclui a avaliação de diversas dimensões ligadas ao comportamento exploratório, tais como nível de satisfação com a informação e estresse associado ao processo de exploração. Contudo, trata-se de um instrumento relativamente extenso que não é facilmente aplicável com outras escalas na realização de pesquisas. Um outro instrumento de exploração vocacional existente em língua portuguesa, com evidências de validade para população universitária brasileira, são as Escalas de Exploração Vocacional (EEV) (Teixeira, Bardagi \& Hutz, 2007), que são instrumentos relativamente curtos, com 24 itens, que medem as dimensões de exploração de si e do ambiente, conforme descrito anteriormente.

Dada a necessidade de instrumentos válidos e fidedignos para realizar pesquisas sobre comportamento exploratório em adolescentes no Brasil, este estudo teve como objetivo testar uma versão adaptada das Escalas de Exploração Vocacional (Teixeira et al., 2007) para 
estudantes de ensino médio. A expectativa foi a de que a estrutura bidimensional do instrumento (exploração de si e do ambiente) se confirmasse empiricamente na versão para estudantes de ensino médio, indicando sua validade de construto. Além disso, pretendeu-se também verificar a fidedignidade das escalas, aferindo sua consistência interna.

\section{Método}

\section{Participantes}

Participaram do estudo 436 estudantes de ensino médio de escolas públicas das cidades de Santa Maria e de Porto Alegre (RS), com média de idade de 16,3 anos (Desvio-Padrão - DP=1,33). As mulheres representaram $55,3 \%$ da amostra. Quanto à série, 47,2\% eram do primeiro ano, 25,7\%, do segundo e 27,1\%, do terceiro. A renda familiar relatada foi de até 2 mil reais para $77,8 \%$ da amostra, entre 2 mil e 4 mil reais para 15,7\% e acima de 4 mil reais para 6,5\%. O nível indicado de escolaridade dos pais foi: primeiro grau incompleto $(38,2 \%$ - pais, $36,8 \%$ - mães), primeiro grau completo (16,2\% - pais, $18,4 \%$ - mães), segundo grau completo (30,5\% - pais, $32,2 \%$ - mães) e terceiro grau completo (14,8\% - pais, $12,4 \%$ - mães). Dos participantes, 0,2\% não soube indicar a escolaridade dos pais.

\section{Instrumento}

O instrumento empregado foi uma versão modificada das Escalas de Exploração Vocacional para universitários (Teixeira et al., 2007), que avalia as dimensões exploração vocacional do ambiente e de si. As modificações introduzidas foram feitas pelos autores sem o uso de juízes, uma vez que não foram consideradas alterações substanciais de conteúdo (buscou-se, é claro, manter a coerência entre as definições dos construtos e seus indicadores comportamentais presentes nos itens). Os itens modificados, em sua maioria, tiveram ligeiras alterações na forma de redação a fim de adequá-los ao tipo de experiências exploratórias esperadas para adolescentes (por exemplo, o item "Quando procuro informações sobre uma atividade, eu também busco descobrir quais são os seus possíveis aspectos negativos", da versão universitária, foi modificado para
"Quando procuro informações sobre uma profissão, eu também busco descobrir quais são os seus possíveis aspectos negativos").

Dos 14 itens de exploração do ambiente da versão universitária, quatro foram mantidos sem alteração alguma, sete sofreram pequenas modificações e três foram eliminados por serem considerados difíceis de serem respondidos pelos adolescentes ou não adequados às suas experiências de vida ("Eu tenho buscado oportunidades para exercitar as habilidades referentes à minha profissão"). Um novo item foi criado ("Eu procuro conversar com pessoas que trabalham em profissões que me atraem para saber mais sobre elas"), ficando a escala de exploração do ambiente composta por 12 itens.

Todos os 10 itens de exploração de si da versão original foram mantidos sem alteração nenhuma, e dois itens foram acrescentados ("Eu tento me imaginar trabalhando em várias profissões para ver como eu me sinto nelas" e "Eu busco distinguir o que eu realmente quero para mim (em termos profissionais) das influências que eu tenho recebido de parentes ou amigos"), resultando em uma escala de 12 itens também.

As seguintes instruções foram apresentadas impressas: "Abaixo há uma série de frases que descrevem atitudes e comportamentos pessoais referentes à escolha profissional. Avalie com que frequência você se envolveu ou tem se envolvido em cada uma das situações listadas, de acordo com a chave de respostas dada. Para responder às questões considere seu comportamento e suas atitudes durante os últimos seis meses". Em seguida, entre as instruções e os itens, foi apresentada a chave de respostas: 1) Raramente ou nunca; 2) Poucas vezes; 3) Com alguma frequência; 4) Frequentemente; 5) Muito frequentemente ou sempre.

\section{Procedimentos}

As escolas foram escolhidas por conveniência. Uma vez obtida a permissão para a pesquisa, foi feito o agendamento da aplicação dos questionários, conforme disponibilidade. Anteriormente ao dia da aplicação dos instrumentos, os alunos foram informados, em sala de aula, sobre a proposta da pesquisa e receberam termos de consentimento para serem assinados pelos pais e 
por eles e trazidos no dia da realização da pesquisa. A aplicação foi feita coletivamente em sala de aula. A pesquisa foi previamente aprovada, em 3/9/2007 pelo Comitê de Ética em Pesquisa da Universidade Federal do Rio Grande do Sul, protocolo n 07/019.

Em relação às Escalas de Exploração Vocacional, foram realizadas análises de componentes principais para verificar a estrutura de componentes do instrumento, bem como análises de consistência interna e correlações item-restante. Posteriormente, dados descritivos (Médias - Me Desvios-Padrão - DP) foram obtidos para as variáveis. Diferenças entre os sexos e entre os diferentes anos do ensino médio foram investigadas por meio do teste te análises de variância.

Tabela 1. Estrutura componencial dos itens das escalas de exploração vocacional para adolescentes. Santa Maria e Porto Alegre (RS), $2007-2008$.

\begin{tabular}{lll}
\hline Itens & Componentes \\
& $\|$
\end{tabular}

1 Quando ouço falar sobre uma nova atividade profissional que me chama a atenção eu procuro mais informações sobre 0,56 ela.

2 Costumo ler livros (ou revistas), assistir a programas de TV (ou vídeos) ou procurar páginas na Internet que trazem 0,43 informações sobre profissões.

3 Eu tenho visitado locais de trabalho para conhecer de perto o dia a dia de profissões que me chamam a atenção. $\quad 0,54$

4 Eu converso com meus pais, professores ou amigos para conhecer mais sobre as possibilidades profissionais que 0,37 existem.

5 Eu tenho buscado obter informações sobre o mercado de trabalho e oportunidades de emprego nas áreas profissionais $\quad 0,58$ de minha preferência.

6 Quando procuro informações sobre uma profissão, eu também busco descobrir quais são os seus possíveis aspectos 0,63 negativos.

7 Eu procuro conversar com pessoas que trabalham em profissões que me atraem para saber mais sobre elas. $\quad$ 0,69

8 Eu tenho procurado conhecer as diversas possibilidades de atuação profissional que existem nas profissões que me 0,65 interessam (coisas diferentes que um mesmo profissional pode fazer).

9 Eu tenho buscado informações sobre quanto ganham realmente os profissionais que atuam nas áreas que me 0,49 interessam.

10 Eu procuro conhecer as possibilidades de crescimento profissional que existem nas profissões que me atraem. $\quad 0,57$

11 Eu tenho procurado me informar sobre as principais dificuldades encontradas pelos profissionais das minhas áreas de 0,59 interesse.

12 Eu tenho tentado conhecer o máximo que eu posso sobre as diversas atividades profissionais que existem na atualidade. 0,43

13 Eu tenho parado para pensar sobre que tipos de atividades profissionais realmente me interessam. $-0,62$

14 Eu tento me imaginar trabalhando em várias profissões para ver como eu me sinto nelas.

15 Eu me coloco em situações que são novas para mim com o objetivo de me conhecermelhor através de experiências $\quad-0,42$ diferentes.

16 Costumo pensar sobre quais são minhas principais habilidades e limitações.

17 Tenho avaliado meus interesses e preferências profissionais. $\quad-0,42$

18 Eu tenho pensado na forma como o meu passado se relaciona com a minha escolha profissional. $\quad-0,43$

19 Eu busco refletir sobre as minhas experiências pessoais para aprender mais sobre mim mesmo.

20 Eu tenho me questionado sobre o que eu realmente considero importante em uma profissão.

21 Eu tenho refletido sobre minha história pessoal quando penso sobre o meu futuro profissional.

22 Eu tenho pensado sobre como o meu jeito de ser pode estar relacionado com as minhas preferências profissionais.

23 Eu busco distinguir o que eu realmente quero para mim (em termos profissionais) das influências que eu tenho recebido $\quad-0,54$ de parentes ou amigos.

24 Eu tenho refletido sobre como as habilidades que eu tenho combinam com as atividades profissionais que me interessam.

92 Nota: são exibidas apenas as cargas de valor superior a 0,30. 


\section{Resultados}

Para verificar a estrutura de componentes subjacente ao conjunto dos itens, foi realizada uma análise de componentes principais, utilizando o critério de autovalor maior do que 1 para a retenção de componentes, e empregando o método oblimin de rotação. $\mathrm{O}$ indicador Kayser-Meyer-Olkin (KMO) obtido foi de 0,88, e o teste de esfericidade de Bartlett mostrou-se significativo ( $p<0,001)$, sugerindo a adequação da análise. Seis componentes apresentaram autovalores maiores do que 1 , explicando 54,4\% da variação total. Porém, a interpretação dos componentes ficou ambígua. Uma análise da declividade dos autovalores indicou que soluções de três, dois ou mesmo um componente poderiam ser mais adequadas. Em função disso, testou-se primeiramente uma solução retendo três componentes para rotação (explicando 40,5\% da variação). Essa solução, contudo, apresentou um componente para o qual não foi possível atribuir um significado consistente.

Em seguida, testou-se a solução de dois componentes, que era esperada teoricamente. Esses dois componentes explicaram 34,5\% da variação total (27\% e 7,5\% para o primeiro e o segundo componente, respectivamente). A Tabela 1 mostra os resultados da análise de componentes principais. Todos os itens elaborados para avaliar exploração do ambiente (itens 1 a 12) apresentaram cargas mais elevadas (acima de 0,35 ) no primeiro componente, enquanto os demais (itens 13 a 24), construídos para medir exploração de si, carregaram mais fortemente no segundo componente. As comunalidades variaram de 0,21 a 0,49. Considerou-se esta a melhor solução por ser teoricamente consistente e compatível com as expectativas.

Analisaram-se, em seguida, as correlações item-restante e os índices de consistência interna de cada escala (alpha de Cronbach). Para a escala de exploração do ambiente, as correlações item-restante variaram de 0,31 a 0,54, e o al pha observado foi de 0,81. Já para a escala de exploração de si as correlações item-restante ficaram entre 0,35 e 0,58, tendo-se obtido um alpha de 0,83.

A partir desses resultados, foram computados escores para cada escala, calculando-se a média das respostas dadas a cada item (os escores mínimo e máximo possíveis são, portanto, 1 e 5, correspondendo ao somatório das respostas dividido por 12). Os itens de 1 a 12 compõem a escala de exploração do ambiente, e os de 13 a 24, a escala de exploração de si. Uma análise de correlação indicou uma relação positiva e significativa entre exploração de si e do ambiente $(r=0,58 ; p<0,001)$. Não foram observadas correlações significativas entre idade e exploração do ambiente $(r=0,07 ; p=0,162)$ nem entre idade e exploração de si $(r=-0,08 ; p=0,113)$. (Tabela 2).

A fim de verificar possíveis diferenças entre homens e mulheres, bem como entre as séries do ensino médio, foram realizados testes $t$ e análises de variância. Os testes $t$ mostraram uma diferença estatisticamente significativa entre os sexos para exploração de si $[t(434)=3,31 ; p=0,001]$, mas não para exploração do ambiente $[t(434)=1,69 ; p=0,092]$. Contudo, o tamanho do efeito observado nessas comparações foi pequeno (valores aproximados: $d=0,16$ para exploração do ambiente e $d=0,32$ para exploração de si; Cohen, 1992).

Já em relação às séries, verificaram-se diferenças estatisticamente significativas tanto em exploração do ambiente $[F(2,433)=5,68 ; p=0,004]$ quanto em exploração de si $[F(2,433)=7,63 ; p=0,001]$. Testes post-hoc (Tukey HSD) mostraram que o escore do grupo do terceiro ano foi mais alto que o do segundo ano em exploração do ambiente, enquanto o escore do grupo do primeiro ano foi mais alto do que o do segundo ano em exploração de si. O tamanho dessas duas diferenças, porém,

Tabela 2. Médias e desvios-padrão das escalas de exploração vocacional para adolescentes. Santa Maria e Porto Alegre (RS), 2007-2008.

\begin{tabular}{|c|c|c|c|c|c|c|}
\hline \multirow{2}{*}{ Variável/Série } & \multicolumn{2}{|c|}{ Homens } & \multicolumn{2}{|c|}{ Mulheres } & \multicolumn{2}{|c|}{ Total } \\
\hline & $M$ & DP & $M$ & $\mathrm{DP}$ & M & DP \\
\hline \multicolumn{7}{|c|}{ Exploração do ambiente } \\
\hline Primeiro ano & 2,90 & 0,74 & 2,98 & 0,67 & 2,95 & 0,70 \\
\hline Segundo ano & 2,70 & 0,64 & 2,80 & 0,82 & 2,76 & 0,75 \\
\hline Terceiro ano & 2,94 & 0,73 & 3,24 & 0,77 & 3,08 & 0,76 \\
\hline Total & 2,87 & 0,72 & 2,99 & 0,75 & 2,93 & 0,74 \\
\hline \multicolumn{7}{|c|}{ Exploração de si } \\
\hline Primeiro ano & 3,20 & 0,68 & 3,66 & 0,70 & 3,46 & 0,73 \\
\hline Segundo ano & 3,18 & 0,75 & 3,09 & 0,78 & 3,13 & 0,76 \\
\hline Terceiro ano & 3,21 & 0,76 & 3,39 & 0,80 & 3,29 & 0,78 \\
\hline Total & 3,20 & 0,72 & 3,44 & 0,78 & 3,33 & 0,76 \\
\hline
\end{tabular}

M: médias; DP: desvios-padrão. 
também não foi elevado, embora mais próximo do que pode ser considerado um efeito de tamanho médio (valores aproximados: $d=0,42$ para exploração do ambiente e $d=0,44$ para exploração de si; Cohen, 1992).

\section{Discussão}

O objetivo principal desta pesquisa foi verificar se a estrutura de duas dimensões da exploração vocacional (do ambiente e de si) emergiria empiricamente da análise das respostas dadas aos itens das Escalas de Exploração Vocacional para estudantes de ensino médio, conferindo assim evidência de validade de construto às escalas. Os resultados obtidos com a análise de componentes principais indicaram, conforme esperado, a existência de duas dimensões subjacentes, com todos os itens carregando de acordo com as expectativas (ou seja, com cargas elevadas em um componente, e baixas em outro). Portanto, o instrumento testado neste estudo apresenta validade de construto no que diz respeito à sua estrutura dimensional.

A pertinência dos itens em cada uma das escalas também pôde ser verificada através das análises de correlação item-restante. Em ambas as escalas as correlações observadas foram positivas e dentro de uma amplitude aceitável (mínima de 0,31 e máxima de 0,58), indicando a coerência do conjunto dos itens. Os alphas de Cronbach obtidos, ambos superiores a 0,80, revelam que as escalas possuem boa fidedignidade.

Apesar de serem aspectos diferentes da exploração vocacional, as dimensões exploração do ambiente e exploração de si mostraram-se correlacionadas. Conforme apontado na introdução, tal resultado faz sentido teoricamente, já que a busca por informações sobre o mundo ocupacional possivelmente leve o indivíduo a refletir sobre suas preferências e interesses e a indagar-se sobre suas habilidades, valores e personalidade. Do mesmo modo, a autorreflexão acerca das características pessoais com vistas a uma decisão vocacional pode produzir a necessidade de busca de mais informações sobre o mundo do trabalho. Essa associação entre exploração de si e do ambiente é também consistente com os resultados obtidos com a versão para universitários das EEV (Teixeira et al., 2007), bem como com outras pesquisas realizadas com adolescentes (Frischenbruder, 1999; Sparta, 2003; Taveira, 2000).

Do mesmo modo, as diferenças de gênero observadas neste estudo são compatíveis com outros estudos realizados com adolescentes, que revelam uma tendência de as mulheres apresentarem maior intensidade ou frequência em comportamentos exploratórios do que os homens (Frischenbruder, 1999; Sparta, 2003; Taveira, 2000; Vignoli, Croity-Belz, Chapeland, Fillipis \& Garcia, 2005). Embora a diferença em exploração do ambiente não tenha sido estatisticamente significativa no nível de 5\%, o valor p observado foi inferior a 0,10, sugerindo que a diferença possa mesmo existir na população. Porém, deve-se ter claro que o tamanho das diferenças observadas é pequeno, tanto em exploração de si quanto do ambiente, sendo talvez irrelevante do ponto de vista prático.

É preciso destacar ainda que nem todos os estudos têm encontrado diferenças de gênero em exploração (Ketterson \& Blustein, 1997; Teixeira et al., 2007). É possível que as pequenas diferenças observadas nos estudos (quando existem) reflitam mais uma maior preocupação e comprometimento das mulheres em relação às questões de decisões vocacionais (Sparta, 2003; Neiva, 2003) do que propriamente um maior engajamento em comportamentos exploratórios. Essa hipótese é coerente com o fato de que a diferença mais saliente observada foi na dimensão de exploração de si, relacionada com a autorreflexão, enquanto na exploração do ambiente a diferença foi ainda menor.

No que concerne às diferenças verificadas entre as séries do ensino médio, os resultados são de difícil interpretação. Seria plausível esperar um aumento no comportamento exploratório ao longo dos anos, mesmo que pequeno, à medida que o fim do ensino médio e a transição para o trabalho ou ensino superior se aproximam, provocando um maior questionamento diante da necessidade de uma tomada de decisão (Joordan, 1963). Contudo, observou-se uma maior exploração de si mesmo nos alunos de primeiro ano quando comparados aos de segundo ano, e uma maior exploração do ambiente em alunos do terceiro quando comparados aos do segundo. É possível que, no primeiro ano, os alunos estejam de algum modo mais propensos a refletir sobre suas características pessoais e preferências 
profissionais em virtude de terem passado mais recentemente por uma transição educacional (do ensino fundamental para o médio), o que pode funcionar como um evento disparador desse tipo de reflexão. Tal tipo de exploração, contudo, pode diminuir no segundo ano, voltando a aumentar no terceiro ano quando o fim do ensino médio se aproxima. A diferença observada em exploração do ambiente, maior no grupo de terceiro ano quando comparada à do grupo de segundo ano, é coerente com a ideia de que, diante da necessidade de uma decisão em relação ao futuro ocupacional, os jovens se envolvam em comportamentos capazes de ampliar seu conhecimento sobre o mundo do trabalho e as opções educacionais existentes.

Todavia, é preciso considerar que o envolvimento dos estudantes em comportamentos exploratórios (de si ou do ambiente) depende em boa parte do quanto estão motivados para isso, ou seja, do quanto se percebem preocupados com a questão vocacional em um dado momento. Jovens que não veem o papel profissional como algo central em sua definição de identidade pessoal talvez explorem menos do que os outros. Da mesma forma, aqueles que já tomaram uma decisão vocacional talvez não sintam mais necessidade de explorar. Assim, o significado do nível de comportamento exploratório vocacional do adolescente precisa ser contextualizado levando-se em consideração seu desenvolvimento vocacional de modo mais amplo (se está preocupado com a questão vocacional, qual a importância atribuída a essa questão, a clareza que possui dos seus objetivos profissionais, a quantidade e a qualidade do conhecimento que já possui sobre as opções educacionais e de trabalho etc.).

Enfim, este estudo mostrou que as EEV para estudantes de ensino médio são um instrumento válido - ao menos no que se relaciona à sua estrutura dimensional - e fidedigno para avaliar comportamentos exploratórios vocacionais com estudantes de ensino médio. Outras pesquisas, contudo, são necessárias para ampliar as evidências de validade do instrumento e também para avançar a teorização sobre desenvolvimento vocacional, mais especificamente no que diz respeito à exploração. Os resultados obtidos nesta pesquisa mostram, empiricamente, que é possível distinguir exploração vocacional de si e do ambiente; porém os fatores que fazem com que os adolescentes difiram entre si nessas variáveis ainda não são claros. Do mesmo modo, é preciso investigar de que forma a exploração vocacional se relaciona com outros indicadores de desenvolvimento vocacional, como clareza de autoconceito profissional, nível de (in)decisão e sentimento de eficácia para a tomada de decisão. Apesar da necessidade de estudos adicionais, as EEV parecem ser um instrumento adequado para pesquisas sobre o tema, podendo futuramente ser utilizadas em contextos aplicados de orientação profissional.

\section{Referências}

Cohen, J. (1992). A power primer. Psychological Bulletin, 112, 155-159.

Flum, H., \& Blustein, D. L. (2000). Reinvigorating the study of vocational exploration: a framework for research. Journal of Vocational Behavior, 56, 380-404.

Frischenbruder, S. L. (1999). O desenvolvimento vocacional na adolescência: autoconceito e comportamento exploratório. Dissertação de mestrado não-publicada, Pontifícia Universidade Católica do Rio Grande do Sul, Porto Alegre.

Hirschi, A., \& Läge, D. (2008). Increasing the career choice readiness of young adolescents: an evaluation study. International Journal of Vocational and Educational Guidance, 8, 95-110.

Jepsen, D. A., \& Dickson, G. L. (2003). Continuity in life-span career development. Career Development Quarterly, 51, 217-233.

Jordaan, J. P. (1963). Exploratory behavior: The formation of selfand occupational concepts. In D. Super, R. Starishevsky, N. Matlin \& J. P. Jordaan (Orgs.), Career development: self-concept theory: essays in vocational development (pp.42-78). New York: College Entrance Examination Board.

Ketterson, T. U., \& Blustein, D. L. (1997). Attachment relationships and the career exploration process. The Career Development Quarterly, 46, 167-178.

Magalhães, M. O., Lassance, M. C., \& Gomes, W. B. (1998). Perspectiva experiencial da indecisão vocacional em adolescentes. Revista da ABOP, 2 (1), 21-58.

Neiva, K. M. C. (2003). A maturidade para a escolha profissional: uma comparação entre alunos do ensino médio. Revista Brasileira de Orientação Profissional, 4, 97-103.

Reed, M. B., Bruch, M. A., \& Haase, R. F. (2004). Five-factor model of personality and career exploration. Journal of Career Assessment, 12, 223-238.

Rogers, M. E., Creed, P. A., \& Glendon, A. I. (2008). The role of personality in adolescent career planning and exploration: a social cognitive perspective. Journal of Vocational Behavior, 73, 132-142. 
Scott, A. B., \& Ciani, K. D. (2008). Effects of an undergraduate career class on men's and women's career decisionmaking self-efficacy and vocational identity. Journal of Career Development, 34, 263-285.

Sparta, M. (2003). A exploração e a indecisão vocacionais em adolescentes no contexto educacional brasileiro. Dissertação de mestrado não-publicada, Universidade Federal do Rio Grande do Sul, Porto Alegre.

Stumpf, S. A., Colarelli, S. M., \& Hartman, K. (1983). Development of the career exploration survey. Journal of Vocational Behavior, 22, 191-226.

Super, D. E. (1963). Vocational development in adolescence and early adulthood: tasks and behaviors. In D. E. Super, R. Starishevsky, N. Martin \& J. P. Jordaan (Orgs.), Career development: self concept theory - essays in vocational development (pp.79-95). New York: College Entrance Examination Board.

Super, D. E., Savickas, M. L., \& Super, C. M. (1996). The life-span, life-space approach to careers. In D. Brown, L.
Brooks \& Associates (Orgs.), Careerchoice and development (3nd ed., pp.121-178). San Francisco: Jossey-Bass.

Taveira, M. C. (2000). Exploração e desenvolvimento vocacional de jovens: estudo sobre as relações entre a exploração, a identidade e a indecisão vocacional. Braga: Universidade do Minho.

Teixeira, M. A. P., Bardagi, M. P., \& Hutz, C. S. (2007). Escalas de exploração vocacional (EEV) para universitários. Psicologia em Estudo, 12, 195-202.

Vignoli, E., Croity-Belz, S., Chapeland, V., Fillipis, A., \& Garcia, M. (2005). Career exploration in adolescents: the role of anxiety, attachment, and parenting style. Journal of Vocational Behavior, 67, 153-168.

Recebido em: 19/3/2008

Versão final reapresentada em: 23/2/2010

Aprovado em: 13/7/2010 